\title{
Infant mental health home visiting therapists' fidelity to the Michigan IMH-HV model in community practice settings
}

\author{
Alissa C. Huth-Bocks ${ }^{1} \quad$ Jennifer M. Jester ${ }^{2} \quad$ Ann M. Stacks ${ }^{3} \quad$ Maria Muzik ${ }^{2}$ \\ Katherine L. Rosenblum² | the Michigan Collaborative for Infant Mental Health Research*
}

${ }^{1}$ Department of Pediatrics, University Hospitals Cleveland Medical Center, Rainbow Babies and Children's Hospital, School of Medicine, Case Western Reserve University, Cleveland, Ohio

${ }^{2}$ Department of Psychiatry, University of Michigan, Ann Arbor, Michigan

${ }^{3}$ Merrill Palmer Institute, Wayne State University, Detroit, Michigan

Correspondence

Alissa C. Huth-Bocks, Department of Pediatrics, University Hospitals Cleveland Medical Center, Rainbow Babies and Children's Hospital, School of Medicine, Case Western Reserve University, Cleveland, OH 44106.

Email: alissa.huth-bocks@case.edu

*The Michigan Collaborative for Infant Mental Health Research (MCIMHR) is composed of researchers from eight universities and from the Alliance for the Advancement of Infant Mental Health, each of whom has collaborated in the design and implementation of the current study. MCIMHR members include (in alphabetical order): Holly Brophy-Herb, Hiram Fitzgerald, Alissa Huth-Bocks, Jennifer Jester, Megan Julian, Jamie Lawler, Alyssa Meuwissen, Maria Muzik, Larissa Niec, Julie Ribaudo, Katherine L Rosenblum, Sarah Shea, Paul Spicer, Ann Stacks, Laurie Van Egeren, Christopher Watson, and Deborah Weatherston.

\begin{abstract}
Implementation research suggests that fidelity to a therapeutic model is important for enhancing outcomes, yet can be difficult to achieve in community practice settings. Furthermore, few published studies have reported on characteristics of treatment fidelity. The present study examined fidelity to the Infant Mental Health Home Visiting (IMH-HV) model among 51 therapists with a range of experience practicing in community settings across the state of Michigan. IMH therapists completed fidelity checklists after every session with participating families to track use of 15 treatment strategies central to the IMH-HV model across the 12-month study period. Results indicated that the most commonly endorsed components utilized in home visits were developmental guidance and infant-parent psychotherapy, followed by the provision of emotional support. Use of IMH-HV components did not vary over time for the entire sample; however, patterns of strategies used showed somewhat more variability among more experienced therapists and when serving higher risk families. Findings demonstrate that IMH-HV therapists report a range of adherence to the model in community settings, with greatest fidelity to several model core components. Ongoing training in the flexible use of all core strategies may further enhance fidelity and contribute to positive outcomes for caregivers and their children receiving IMH-HV services.
\end{abstract}

\section{K E Y W O R D S}

fidelity, home visiting, implementation, infant mental health

\section{1 | INTRODUCTION}

There is a large science-to-practice gap for evidence-based treatments, particularly when treatments are implemented in community practice settings. There is also a vital need to better evaluate and report on clinician fidelity (i.e., model adherence) to core treatment components of evidence-based therapies. Michigan's Infant Mental Health Home Visiting (IMH-HV) model is a relationship-focused home visiting intervention serving parents and their infants and toddlers across the state in community mental health settings. Rooted firmly in attachment and other relational theories, IMH-HV was initially developed by Selma Fraiberg in the mid-1970s (Fraiberg, 1980) and later formalized over many years by IMH leaders across the state of Michigan (Weatherston \& Tableman, 2015; for a comprehensive description of the model, see Weatherston \& Ribaudo, this issue). The model is flexible and family driven; thus, there is neither a 
predetermined "dose" of the intervention nor a required sequence of intervention strategies. Given the long history of IMH-HV services, as well as the relatively nonstructured, flexible nature of the intervention, ongoing evaluation of this model provides an important opportunity to describe what (and how) it is being delivered and the degree to which community therapists providing IMH-HV services adhere to the model.

Fidelity to a treatment model is one component of implementation that has been associated with positive clinical outcomes; however, fidelity can be difficult to achieve when evidence-based models are disseminated into community practice settings (David \& Schiff, 2018; Durlak \& Dupree, 2008; Hulleman \& Cordray, 2009). The aim of the present study was to examine and describe fidelity to the IMH-HV model among a group of clinicians with a wide range of training and years of experience working within publicly funded community mental health settings. Understanding more about therapist fidelity to the IMH-HV model, particularly to the model's core components, fills an important gap in our current understanding of IMH-HV model implementation, as well as the broader home visiting literature.

\section{1 | Implementation and fidelity to intervention core components in community settings}

Implementation describes the elements of a program in a particular setting (Durlak \& Dupree, 2008), and implementation research involves determining the effectiveness of programs when delivered in settings of community-based practice (Weisz \& Jensen, 1999). Researchers have described eight different aspects of implementation quality, including fidelity or adherence to the program model; dosage; quality of delivery, including the skill with which clinicians interact with participants; participant responsiveness and level of enthusiasm; adaptation; reach; and monitoring of a comparison condition (Berkel, Mauricio, Am, Scoenfelder, \& Sandler, 2011; Dane \& Schneider, 1998). Both fidelity and quality of delivery, specifically, describe the use of essential ingredients of a model (David \& Schiff, 2018; Fixsen, Naoom, Blasé, Friedman \& Wallace, 2005), and both can be supported through ongoing training, technical assistance, and supervision (Durlak \& Dupree, 2008; Fixsen et al, 2005; Roben, Dozier, Caron \& Bernard, 2017). Fidelity or adherence to a model is the degree to which the specified model components are delivered as prescribed, the percentage of manualized content delivered, and/or the amount of time dedicated to each of the core components. Fidelity has a significant positive association with program outcomes, with some data suggesting that only $60 \%$ adherence may be required for positive outcomes (Dane \& Schneider, 1998; Durlak \& Dupree, 2008; Fixsen et al., 2005). Among psychotherapeutic interventions, there is evidence that the quality of delivery, especially relationship factors, is also essential for good outcomes (Berkel et al., 2011).

When evidence-based interventions are moved into the community, the outcomes are often not as robust as those seen in the laboratory, or other controlled settings, due in part to a lack of fidelity to the model (Durlak \& Dupree, 2008; Hulleman \& Cordray, 2009). Although several attachmentoriented, mental health interventions, both home and clinic based (described below), have been shown to be effective in randomized controlled trials, there remains a need to test these interventions in community practice settings. Furthermore, published studies have rarely included findings related to treatment fidelity or mechanisms of change (Suchman et al., 2012). In fact, more broadly, most intervention studies do not document implementation details or information about which aspects of the intervention were delivered, making it difficult to interpret links between treatment components and outcomes (Dane \& Schneider, 1998; Domitrovich \& Greenberg 2000; Perpletchikova, Treat, \& Kazdin, 2007). The larger body of intervention research suggests that programs that do monitor implementation generally have larger effects on outcomes (Dubois, Holloway, Valentine \& Cooper, 2002; Smith, Schneider, Smith \& Ananiadou, 2004). The association between implementation quality and program outcomes is consistent across studies and may account for effect sizes two to three times higher than in those programs not tracking fidelity to a therapeutic model (Derzon, Sale, Springer, \& Brounstein, 2005; Durlak \& Dupree, 2008).

\section{2 | Fidelity in early childhood mental health interventions}

While fidelity and quality of delivery are rarely measured in studies of parenting interventions in early childhood, there are notable exceptions that support the broader implementation findings related to fidelity and program outcomes. For example, the Incredible Years (Webster Stratton, 1990) is an evidence-based group intervention aimed at reducing behavior problems in order to promote social-emotional competence in young children by supporting parents in (a) understanding and assisting with child social skills and emotion regulation, (b) using praise to encourage cooperative behavior, (c) setting limits, and (d) handling misbehavior. Findings from Eames and colleagues (2009) suggest that the quality of program delivery for this intervention model is positively associated with larger improvements in parenting behavior, which in turn predict increases in child positive behaviors.

In an attachment-based intervention more closely related to IMH-HV, the Mothers and Toddlers program (Suchman et al., 2012), research findings have shown that fidelity also supports improvements in the quality of caregiving representations and behavior that are associated with greater 
security in the parent-child relationship. In their randomized controlled pilot study, Suchman and colleagues measured what they termed "generic" core components and "unique" components (referred to as key ingredients in implementation research) thought to specifically improve parents' reflective functioning, representation quality, and caregiving behavior. They operationalized generic core components in parent education programs as including forming a positive therapeutic alliance, providing general developmental guidance, and fostering emotion regulation. The unique core components of their intervention included therapist techniques to foster more positive parental representations of their children, parents' reflective functioning, and therapist use of attachment-based developmental guidance.

Findings from this study suggest that therapist adherence to the key/unique ingredients of the intervention predicted change in representational quality, reflective functioning, and caregiving quality (Suchman et al., 2012). Interestingly, therapists in this study only demonstrated moderate levels of adherence to the key ingredients of the intervention, as assessed by video review of sessions by independent raters. Specifically, video reviews showed that therapists' fidelity to the key components of the model ranged from $35 \%$ to $43 \%$, suggesting that therapists may have practiced more flexibly in order to meet and be responsive to ongoing and immediate client needs that arose during treatment. The findings also highlight that partial fidelity to a therapeutic model in a community practice setting may still lead to beneficial outcomes, such as increases in parental reflective functioning and positive caregiving behavior. These results suggest that flexibility may, in fact, be desirable so long as there is adherence to a model's core components (Kendall \& Frank, 2018); flexibility may even be considered an aspect of fidelity in some models (Lieberman, Ghosh Ippen, \& Van Horn, 2015).

In another attachment-based parenting intervention delivered in a community setting, Attachment and Biobehavioral Catch-up, fidelity to the model's key ingredients was associated with increases in parenting sensitivity and decreases in parent intrusiveness, with effect sizes as large as those seen in trials conducted in laboratory settings (Caron, Bernard, Dozier, 2016; Roben et al., 2017). The key ingredients in this model included therapist in-the-moment comments about specific parenting behavior, links between child behavior and parent behaviors, and comments about how parent behaviors may impact the child. To support clinicians' fidelity to the model, clinicians received weekly group and individual supervision that included video review and feedback on their inthe-moment comments, highlighting the need for significant clinician support to promote model adherence in community practice settings.

Finally, a recently published study (David \& Schiff, 2018) evaluating experienced clinicians' fidelity to Child-Parent Psychotherapy (CPP; Lieberman et al., 2015), a well-known and extensively studied mental health intervention for traumatized caregivers and their young children, demonstrated that clinicians reported very high levels of fidelity to the model's key elements (ranging from $62 \%$ to $99 \%$ for 13 different treatment strategies) in their clinical work. Lowest rates were reported for the administration of trauma-specific questionnaires (62-78\%), whereas very high rates (above 90\%) were reported for all other treatment strategies such as evaluating the safety of the environment, providing psychoeducation, working on emotional regulation, and building a trauma narrative with caregivers and children. However, it is important to note that clinicians were asked to report how often they used each of the strategies "in general" rather than in reference to specific cases, possibly contributing to the very high fidelity rates seen in this study.

\section{3 | The present study}

Taken together, findings from implementation research suggest that clinician fidelity to a therapeutic model is important in predicting outcomes, and several studies have confirmed such associations for early childhood home-based intervention models. Yet, very few published studies have examined and detailed markers of fidelity, despite the known difficulty of achieving treatment fidelity, or model adherence, in community practice settings. The current study aims to describe and evaluate fidelity to the IMH-HV model, a longstanding home-based, mental health intervention, among therapists with a range of training and experience practicing in community mental health settings across the state of Michigan. Because others have found that therapist experience has been linked with fidelity (Berkel et al., 2011), we examined experience as a predictor of fidelity; family risk status was also explored as a potential predictor of model fidelity.

\section{2 | METHOD}

\section{1 | Participants}

Participants in the current study included 51 therapists delivering IMH-HV services to 78 caregivers and their infants and toddlers, as part of an open trial, pre-post design study of the effectiveness of the IMH-HV model on key parenting and child outcomes. Twelve Community Mental Health Service Programs in mid- and southeastern Michigan were identified and partnered with the study team; IMH-HV therapists and their clinical supervisors from these participating sites recruited caregivers (and their children) who had recently initiated services.

All therapists had received a master's degree in social work (63\%) or a related field (37\%), as required by the state's Department of Health and Human Services. Despite universal graduate-level education, therapist experience varied 
T A B L E 1 Therapist-participant characteristics $(N=51)$

\begin{tabular}{lll}
\hline Work experience & Mean $(\boldsymbol{S D})$ or $\%$ & Range \\
\hline Time in IMH field & 43.31 months (42.90 months) & $2-192$ months \\
\hline Time in early childhood field & 66.08 months (66.22 months) & $0-234$ months \\
\hline Current IMH cases & 5.46 cases (2.91 cases) & $1-12$ cases \\
\hline Current total cases & 8.94 cases (3.27 cases) & $3-15$ cases \\
\hline Weekly reflective supervision & $67 \%$ & NA \\
\hline Biweekly reflective supervision & $14 \%$ & NA \\
\hline Monthly reflective supervision & $19 \%$ & NA \\
\hline Waiver for endorsement & $47 \%$ & NA \\
\hline Category II endorsement & $31 \%$ & NA \\
\hline Category III endorsement & $22 \%$ & NA \\
\hline
\end{tabular}

Note. A waiver is required to practice IMH-HV while working toward a Category II or III endorsement.

considerably (see Table 1). Because reflective supervision is an essential core component in the IMH-HV model, all therapists received some form of reflective supervision, provided by an IMH-endorsed clinician-supervisor through their community agency, with the majority receiving weekly supervision. Furthermore, the vast majority (79\%) reported receiving both group and individual reflective supervision, whereas $18 \%$ reported group only and one clinician reported individual supervision only.

Clinicians delivering IMH-HV in the Community Mental Health Services system are required to achieve a Category II (Infant Family Specialist) or Category III (Infant Mental Health Specialist) endorsement by the Michigan Association for Infant Mental Health in Culturally Sensitive, Relationshipfocused Practice Promoting Infant Mental Health ${ }^{\circledR}$ (Michigan Association for Infant Mental Health, 2018). A Waiver of Provider Qualifications to practice IMH-HV is required to practice IMH-HV while working toward Category II or III endorsement. Clinicians in the present study represented several categories of endorsement (see Table 1).

Participating families included both biological $(n=75)$ and/or foster $(n=3)$ mothers $(N=78)$, and their children (mean age $=9.8$ months, $S D=8.4$ months). Average caregiver age was 27 years old ( $S D=6.9$ years). The average total number of people living in the home was $4.6(S D=1.9)$, with an average of $2.3(S D=1.5)$ children living in the home. Families were diverse and represented a range of education levels, marital status, race, and income, although most had never been married and about half of all caregivers had a high school education or less. All children were Medicaid recipients. See Table 2 for detailed family characteristics. As is typical for families receiving Medicaid and IMH-HV services, families were characterized as high-risk due to socioeconomic status, exposure to stress and adversity, and other risk factors. Additionally, about two-thirds of the families reported incomes of less than $\$ 20,000 /$ year. The average number of Adverse Childhood Experiences (ACEs; Felitti et al., 1998) experienced by caregivers was $4.5(S D=3.0)$ out of 10 .
T A B L E 2 Client-participant characteristics $(N=78)$

\begin{tabular}{|lc|}
\hline Family characteristic & Percent (\%) \\
\hline Caregiver race & \\
\hline White/Caucasian & 55 \\
\hline Black/African American & 45 \\
\hline Hispanic/Latina & 5 \\
\hline Other & 5 \\
\hline Caregiver education & \\
\hline Less than high school & 28 \\
\hline High school diploma/General & 26 \\
\hline Education Development (GED) & \\
\hline Some college & 31 \\
\hline Associates/vocational & 10 \\
\hline Bachelor's degree & 5 \\
\hline Marital status & \\
\hline Married & 21 \\
\hline Divorced/separated/widow & 10 \\
\hline Never married & 69 \\
\hline Family income per year & \\
\hline Less than \$10,000 & 49 \\
\hline \$10,000-19,999 & 20 \\
\hline \$20,000-39,999 & 9 \\
\hline \$40,000-79,999 & \\
\hline Percen & \\
\hline
\end{tabular}

Note. Percentage total is $>100 \%$ for caregiver race because participants were allowed to choose more than once race category.

\section{2 | Procedures}

Participating therapists recruited families with young children ages 0-24 months who had recently initiated IMH-HV services. Participating therapists and families both provided written informed consent. Caregivers were incentivized for participation and could receive up to $\$ 280$ total across the study; families completed a variety of measures and tasks at five data collection waves: baseline, 3-, 6-, 9-, and 12-months after baseline. Procedures at baseline, 6-, and 12-months 
T A B L E 3 Infant Mental Health Home Visiting (IMH-HV) treatment strategies on fidelity checklist

\begin{tabular}{|c|c|c|}
\hline IMH-HV strategy & $\begin{array}{l}\text { Number of } \\
\text { example } \\
\text { items }\end{array}$ & Sample item \\
\hline Assessment & 3 & "Assessment of the child/parent relationship using formal assessment tools" \\
\hline Material needs & 3 & $\begin{array}{l}\text { "Helped family obtain material needs including food, housing, supplies, } \\
\text { equipment" }\end{array}$ \\
\hline Health care: Child & 4 & "Attended or facilitated parent attendance at well-child visits" \\
\hline Health care: Parent & 4 & "Identified and facilitated use of PCP" \\
\hline Emotional support & 1 & "Supported parents/family in crisis or life transitions for family" \\
\hline Developmental guidance & 4 & "Interpreted child's behavior and needs from a developmental perspective" \\
\hline Infant-parent psychotherapy & 7 & $\begin{array}{l}\text { "Addressed negative experiences and/or unresolved loss/trauma that may be } \\
\text { affecting relationship with child" }\end{array}$ \\
\hline Life planning & 3 & "Discussed family planning and/or deferral of next birth" \\
\hline Social supports & 1 & "Fostered the development of social supports for/with parent" \\
\hline Special issues: Parent & 1 & $\begin{array}{l}\text { "Addressed issues related to depression, other mental illness, disability, } \\
\text { relational trauma, substance abuse, sexual abuse, pregnancy" }\end{array}$ \\
\hline Special issues: Child & 1 & "Addressed regulatory disorders, health, illness of child" \\
\hline Crisis planning & 2 & "Created safety plan as needed" \\
\hline Environmental safety concerns & 1 & "Addressed issues related to environmental safety" \\
\hline Videotaping & 2 & "Videotaped at home visit" and "Reviewed video with parent" \\
\hline Termination planning & 5 & "Addressed parent and/or child's feelings regarding termination" \\
\hline
\end{tabular}

were collected by trained researchers in the home, whereas a briefer set of measures was administered over the phone by researchers at 3-and 9-months. Therapists attended a 1-day training on data collection and study procedures; for the purposes of the current study, this included several hours of training on study requirements for completing fidelity checklists after each treatment session with a participating family, as well as practice using the checklists. Fidelity forms were submitted by clinicians to the university-based research team. Institutional Review Board (IRB) approval was maintained throughout the duration of the study at the study's home University.

\section{3 | Measures}

\subsection{1 | IMH-HV fidelity}

As noted above, participating IMH-HV therapists completed a fidelity checklist after every session with their participating families. The fidelity checklist used in the present study (Weatherston et al., 2016, available upon request) was an adaptation of Weatherston and Tableman's (2015) Fidelity Tool. The adapted fidelity checklist included 15 broad categories representing IMH-HV strategies; each broad category included multiple items in order to give therapists examples of possible specific interventions within the broad categories (see Table 3). Therapists were asked to check off what they did (all that applied) during each home visit. Emphasis was placed on the broad categories of strategies or interventions; thera- pists were instructed that they could be less specific about the particular items they endorsed having done within the broad categories. As a result, a total of 0-15 broad strategies could have been endorsed. A final item on the fidelity checklist asked clinicians to report the three "most significant" components that were delivered at each visit, with the first component listed as "the component with the most significance."

\subsection{2 | Demographic characteristics and family risk}

A demographics form was completed by caregivers at study entry for characteristics such as age, child gender, race/ethnicity, education level, household income, and relationship status. Various indicators of family risk were also measured through caregiver report in order to create groups based on family risk status for analyses. Demographic risk was measured by young maternal age at childbirth $(<21$ years at time of birth), number of children in the home (4 or more children under 5 years of age), very low income $(<\$ 5000 /$ year), low education (less than high school education), being unmarried, and racial/ethnic minority status. Psychological risk was measured by presence of maternal psychopathology (above established clinical cutoff on measures of depression or posttraumatic stress disorder) and significant childhood adversity (defined as three or more ACEs). Each of the risk factors counted as 1 point for the cumulative risk score; a high risk group (71\%) was defined as 


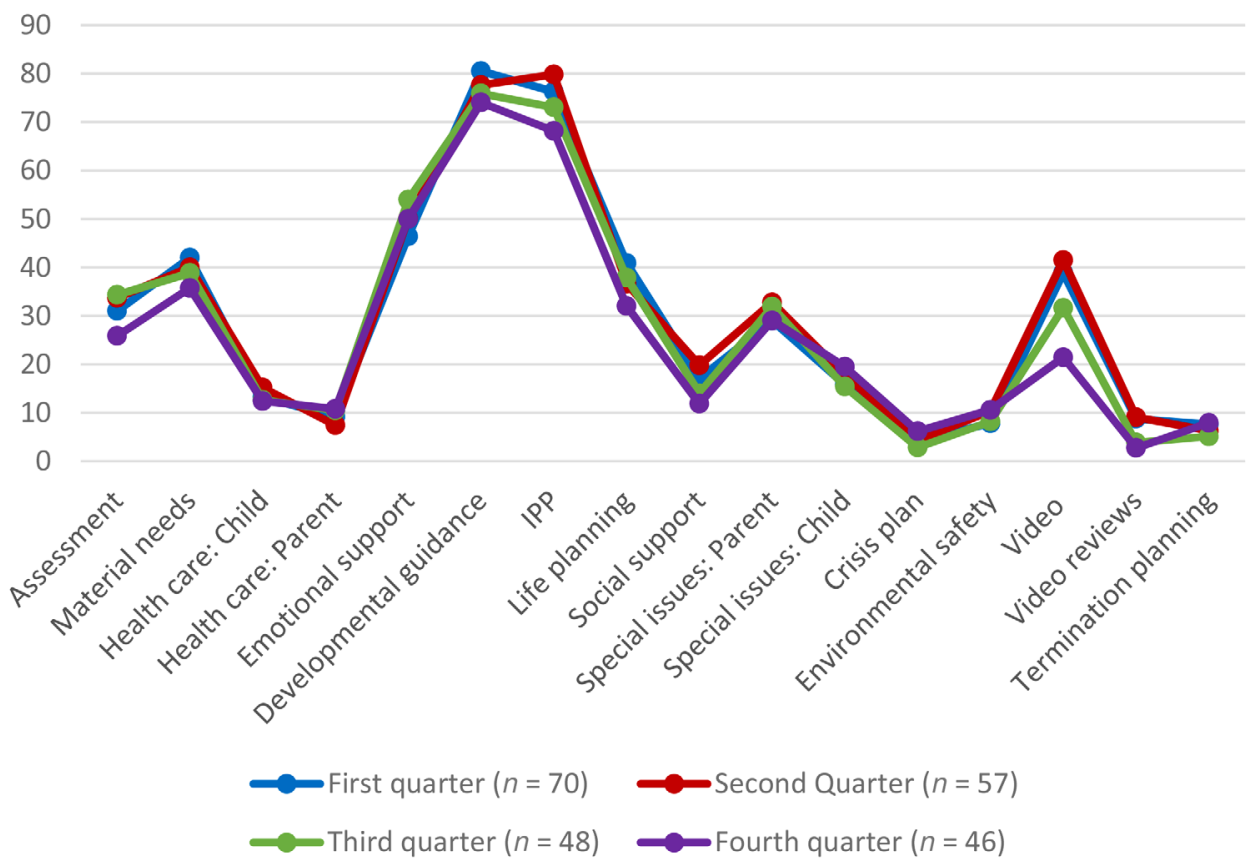

F I G U R E 1 Percent of visits in which therapists endorsed using each component, for all participants with five or more visits in the quarter Note. Rates were also calculated month to month for all participants, which resulted in the same pattern. Therefore, this and all subsequent graphs display results per quarter.

Abbreviation: IPP, infant-parent psychotherapy

a cumulative risk score of 3 or higher and the low risk group (29\%) was defined as a risk score of less than 3.

\subsection{Data analytic plan}

Descriptive statistics were used to calculate means, standard deviations, ranges, percent/proportions, and so forth for variables of interest, namely, therapists' reports of their own utilization of IMH-HV broad intervention strategies in home visit sessions with families. To prepare data on the fidelity checklist, all of the items in each category were first collapsed into the broad category, such that broad categories were endorsed as either happening or not happening in each session (dichotomous variable). Fidelity variables were also grouped into 3-month periods (quarters), so that the percentage of sessions each broad intervention category was endorsed as being used could be calculated (i.e., the number of times that a category was endorsed was totaled for each quarter and divided by the number of treatment sessions in that quarter for each clinician). Only those participants with at least five treatment sessions were included in the calculations of percent of time spent using each category, which are described in the text and displayed in Figures 1-4. This decision was made so that those with a very small number of sessions would not be given undue weight; for example, a participant with a single treatment session who had received one intervention in one category would have been counted as $100 \%$ for that category.
T A B L E 4 Treatment visit totals across duration of Infant Mental Health Home Visiting (IMH-HV) intervention

\begin{tabular}{lcc} 
Treatment visits & Mean $(S D)$ & Range \\
\hline Total, per family & $31.97(17.43)$ & $1-67$ \\
\hline Quarter 1, per family & $11.14(4.35)$ & $1-22$ \\
\hline Quarter 2, per family & $8.59(5.32)$ & $0-19$ \\
\hline Quarter 3, per family & $6.71(5.55)$ & $0-20$ \\
\hline Quarter 4, per family & $5.54(5.34)$ & $0-19$ \\
\hline
\end{tabular}

\section{3 | RESULTS}

The total number of IMH-HV treatment visits reported by therapists in the study over the 12-month study period was 2,568. Twenty-five percent of the sample received 19 or fewer visits, $15 \%$ received 48 or more visits, whereas the majority (60\%) received 20-47 visits. Total number of visits per family, as well as number of visits for each quarter, is shown in Table 4. These data show that the average number of visits decreased substantially across the year. The majority of sessions took place in the family home (82.5\%), whereas $15 \%$ occurred in other locations or on the phone and $2.5 \%$ of sessions occurred in both the home and in another location. The median length of visits was $90 \mathrm{~min}$, with comparable session lengths in all quarters. Finally, therapists reported engaging in 4.5 broad categories of IMH-HV intervention strategies, on average, per session; this number decreased only slightly 


\section{Percent of visits in which the category is top component}

30

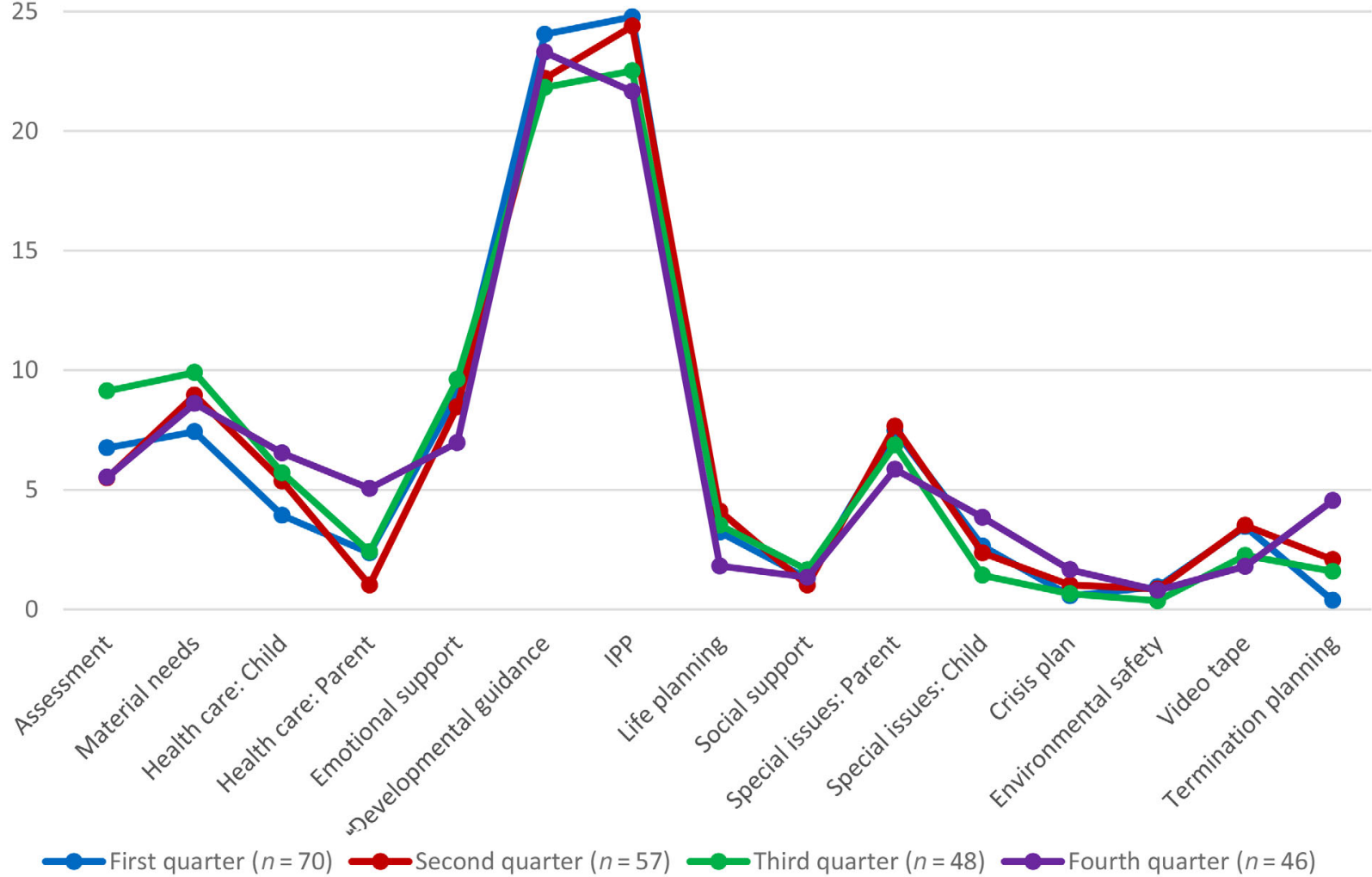

F I G U R E 2 Percent of visits in which therapists endorsed components as most significant during sessions

Abbreviation: IPP, infant-parent psychotherapy

across the year ( 4.6 categories in the first quarter to 4.1 categories in the last quarter).

Figure 1 shows the percentage of treatment visits during which each broad category of IMH-HV treatment components was provided by therapists. Percentages are plotted separately for each quarter of the study year. The most common intervention strategies were developmental guidance and infant-parent psychotherapy. In the first quarter, $81 \%$ of visits included developmental guidance and $76 \%$ included infant-parent psychotherapy. These two core components remained the most frequently utilized treatment strategies over the entire year of the study with only a small decrease for use of developmental guidance to $74 \%$ in the fourth quarter and a slightly larger decrease for use of infant-parent psychotherapy to $68 \%$ in the fourth quarter. Emotional support was the next most commonly endorsed IMH-HV intervention strategy, with use ranging between $46 \%$ and $54 \%$ across quarters. Several components were endorsed as being utilized about one-third of the time including assessment, addressing material needs, life planning, special concerns about the parent, and use of video to capture interactions in the home.
Finally, several core IMH-HV components were infrequently endorsed as being used; these included health care problems with the child and/or parent, assistance with social supports, special issues about the child, crisis planning, addressing environmental safety, video feedback review with the family, and termination planning. Interestingly, the overall shape of the distribution remained quite consistent over the four quarters. That is, the percentage or proportion of visits where the core intervention strategies were or were not used by therapists did not differ considerably by time (i.e., 3-month periods across treatment), with one possible exception. Therapist use of video in the home (but not video feedback with the family) showed more variability across quarters, with rates decreasing from $40 \%$ in the first quarter to $22 \%$ in the fourth quarter.

As noted earlier, the final item on the fidelity checklist asked therapists to list the three most significant components that were provided at each visit, with the first component indicated as the most important intervention according to the therapist. The percent of visits that each component, or broad category, of intervention was listed as the most significant is shown in Figure 2. Similar to utilization patterns in 
general, therapists most commonly rated developmental guidance and infant-parent psychotherapy as the most significant interventions that they provided to families during home visits. These rates were fairly consistent across quarters, although therapists' ratings of infant-parent psychotherapy as the most important intervention decreased slightly from $24 \%$ in the first and second quarters to $22 \%$ in the third and fourth quarters. Rates of developmental guidance as the most important intervention ranged between $22 \%$ and $24 \%$ across the four quarters. All other components were rated as the most significant intervention during a session at some point in the study period, but at substantially lower rates $(0.4 \%$ to $10 \%)$ than the former two IMH-HV core components. Similarly, developmental guidance and infant-parent psychotherapy were listed in the top three most significant interventions at much higher rates than all other components (data not shown); developmental guidance was listed in the top three for $65 \%$ of visits during the first quarter with only a slight drop to $58 \%$ in the fourth quarter, whereas infant-parent psychotherapy was listed in the top three components between $59 \%$ and $63 \%$ of visits across the 12-month study period. Rates of all other components in the top three most significant interventions were much lower and followed the same pattern across quarters as that displayed in Figure 2.

In order to better understand possible variables that may influence fidelity to the IMH-HV model, therapist experience and family risk status were both examined in relation to use of IMH-HV treatment strategies during the study period. Participating caregivers (some of whom had the same clinicians) were first split into two groups: those with therapists who had 5 or more years of experience in the IMH field $(n=21)$ and those with therapists who had less than 5 years of IMH experience $(n=57)$; see Figure 3. Less experienced therapists tended to do more developmental guidance, hovering around $80 \%$ of visits throughout the year, whereas more experienced therapists reported providing about the same rate of developmental guidance initially, but somewhat less developmental guidance (about 69\%) after the second quarter. Less experienced therapists also reported more infant-parent psychotherapy, with rates between $77 \%$ and $82 \%$ across the year, whereas more experienced therapists reported slightly lower rates (between $64 \%$ and $76 \%$ of visits) in the first three quarters, with a drop to $48 \%$ in the last quarter. Less experienced therapists also reported more visits during which they did assessment, ranging from $33 \%$ to $42 \%$ over the year, whereas more experienced therapists reported a gradual decline in assessment from $23 \%$ of visits in the first quarter to $12 \%$ in the fourth quarter. Termination planning and use of videotaping in the home also showed different patterns in more and less experienced therapists. For less experienced therapists, there was a gradual decrease in termination planning from $10 \%$ of visits in the first quarter to $7 \%$ in the fourth quarter. In contrast, experienced therapists had few sessions with termination planning

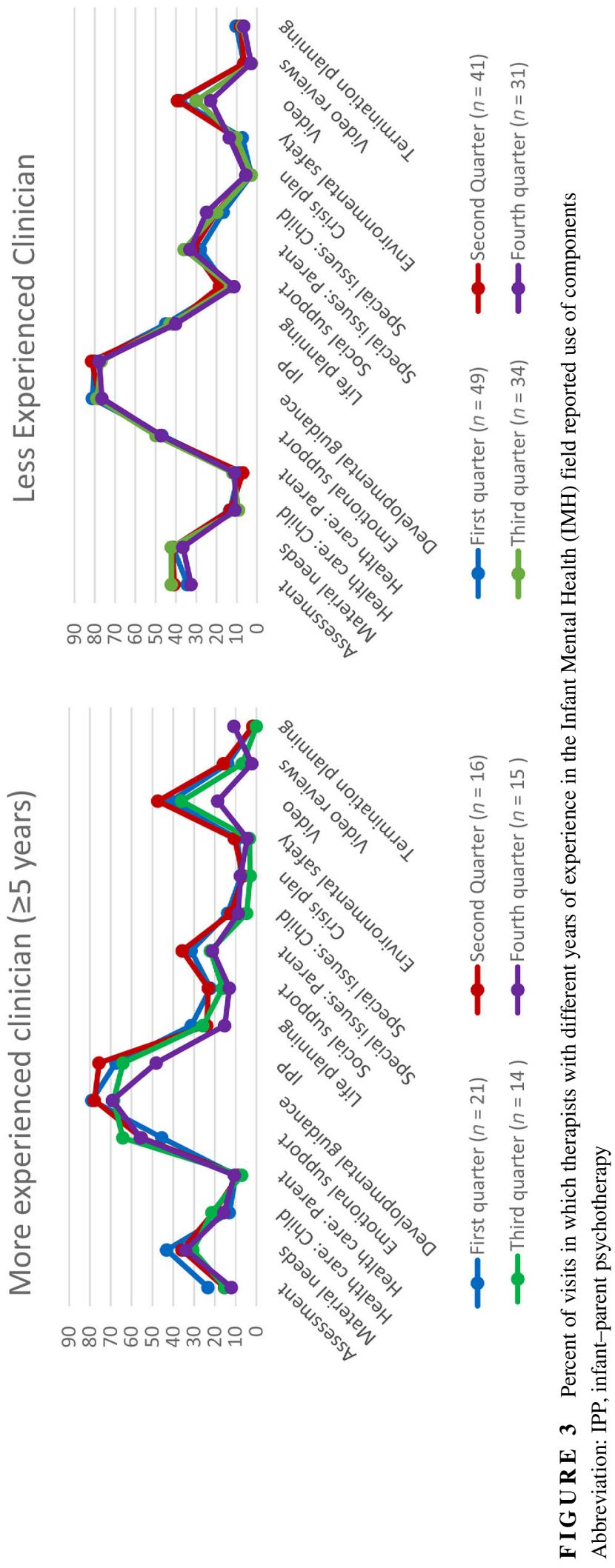


in the first three quarters $(0-2 \%)$, but then reported a notable increase (albeit still small percentage) in termination planning in the last quarter (11\%). In terms of using videotaping in the home, less experienced therapists started out using it in about $39 \%$ of visits across the first two quarters but decreased use to $23 \%$ in the last quarter. In contrast, more experienced therapists used videotaping between $41 \%$ and $47 \%$ of the time across the first three quarters, with a reduction to $19 \%$ in the last quarter; more experienced therapists also reported more video review with the family early in therapy compared to less experienced therapists, although still at relatively low rates. Overall, more experienced therapists tended to report more variability in use of IMH-HV treatment strategies within and across quarters as compared to less experienced therapists.

Finally, rates of therapist use of IMH-HV treatment strategies were examined separately for families defined as lower risk (i.e., those with two or fewer measured risk variables; $n$ $=23$ ) and higher risk (i.e., those with three or more measured risk variables; $n=55$; see Figure 4). Results indicated that, although the overall "pattern" of use for the different IMHHV components was similar in the two groups (and consistent with patterns shown in prior graphs for the whole sample and clinicians with varying levels of training and experience), there was notably more variability of strategies used across quarters when therapists were treating higher risk families. For example, therapists reported a drop in use of infant-parent psychotherapy from $70 \%$ in the first quarter to $48 \%$ in the fourth quarter when treating higher risk families. Similarly, use of developmental guidance started very high at $81 \%$ in the first quarter and dropped to $62 \%$ in the fourth quarter. In contrast, rates for both of these commonly endorsed IMH-HV core components stayed uniformly high across quarters when clinicians were treating lower risk families. Rates for all other components, except assessment, crisis planning, and videotaping in the home, were also very uniform across the study period for lower risk families. For lower risk families, assessment use varied slightly across quarters (but still stayed within a $28-42 \%$ range), as did use of video (dropping from 36-39\% in the first two quarters to $21 \%$ in the last quarter). Interestingly, crisis planning more than doubled in the last quarter (8\%) compared to the first three quarters (2-4\%), although was still infrequently used with lower risk families. For higher risk families, notable variability was observed for most intervention strategies over time including for use of assessment, focus on material needs, focus on life planning, assistance with obtaining social support, addressing special issues about the parent, addressing environmental safety, and use of videotaping. One final observation of note was that rates of virtually all intervention components were lowest in the fourth quarter as compared to earlier in treatment with higher-risk families.

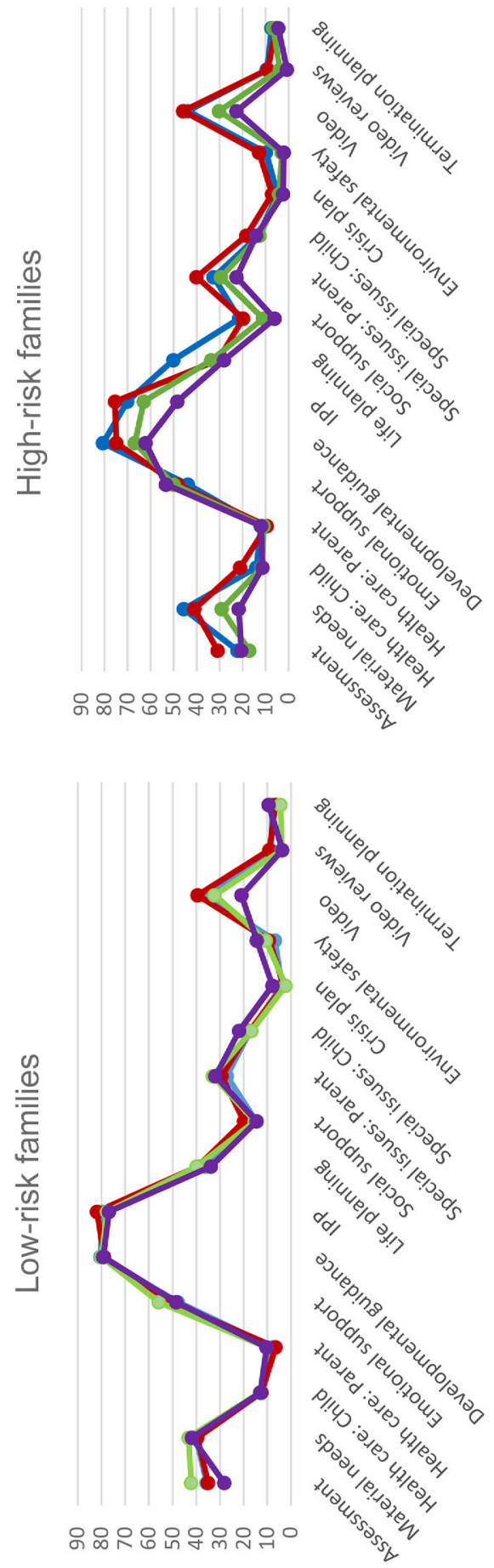




\section{4 | DISCUSSION}

The current study sets out to examine treatment fidelity to the IMH-HV model among therapists practicing in community mental health settings across the state of Michigan. Over many years since its inception, the IMH-HV model has been formalized, competencies have been developed and linked with categories of endorsement for which IMH therapists are required to attain and sustain, and the model is administered throughout the state with services reimbursed by Medicaid. Thus, it is imperative and timely to describe therapist fidelity to this model in order to better understand community therapists' use of the model's core components and treatment strategies.

Overall, results from the present study revealed both expected and unexpected findings. Not surprisingly, IMH-HV therapists at varying levels of training and experience reported using developmental guidance and infant-parent psychotherapy most frequently throughout the duration of treatment with families. These interventions are two of the model's identified core components (see Weatherston \& Ribaudo, this issue), and thus, IMH-HV therapists likely felt both familiar with and confident in using these intervention strategies with families. It is important to remind the reader that all therapists in the study received reflective supervision from the agency at which they were employed, with the vast majority receiving both group and individual reflective supervision. Studies show that the provision of reflective supervision is a predictor of program effectiveness, perhaps because it supports reflective practice skills, which in turn supports implementation quality (Beam, O’Brien, \& Neil, 2010; Casillas, Fauchier, Derkash \& Garrido, 2016; Shea et al., 2020). Thus, it is quite possible that developmental guidance and infant-parent psychotherapy techniques were also often attended to in supervision. Both of these speculations are supported by the additional finding that these two core components of the IMH-HV model were rated much more frequently as the "most significant" (or in the top three most significant) interventions used in sessions. The consistently high levels of provision of emotional support (the third most common component endorsed by therapists) are also not surprising given the necessity of such support in high-need families and the importance of providing emotional support for sustaining a trusting therapeutic relationship. Indeed, others have noted that provision of emotional support is foundational to good practice across several parenting and early childhood interventions, yet alone may not support improvements in parents' representation of their child, reflective capacity, sensitivity, or ultimately child attachment (Lieberman et al., 2015; Suchman et al., 2012).

At first glance, for some, it may be somewhat surprising to see the equally high level of infant-parent psychotherapy early in the treatment as compared to later in the treatment, given that infant-parent psychotherapy often involves deeper exploration into the parent's history and experiences of trauma and loss ("ghosts in the nursery"; Fraiberg, 1980), which requires the establishment of a safe, trusting therapeutic relationship. There are at least two possible explanations for this finding. First, in the present study, infant-parent psychotherapy was operationalized to include not only deep exploration of the mother's past experiences, but also important clinical strategies such as discussing the parent's perceptions and representations of the child, as well as exploring the parent's feelings about the child currently. These options on the fidelity form filled out by therapists after each clinical session may have contributed to the high reported use of infant-parent psychotherapy early in the treatment process; because therapists were asked to endorse the use of broad categories only (and not specific items within each category), this speculation cannot be tested. As such, and because categories were broad, perhaps especially so for infant-parent psychotherapy, it is important to be cautious about this particular finding. Another possibility for the high levels of endorsed use of infant-parent psychotherapy across treatment is that, for some families, the need to address the mother's past experiences, or "ghosts," may have presented early in the treatment, and therapists felt it was important to engage in this work as it emerged. This would be consistent with the IMH-HV flexible, family-driven model.

Results also revealed that therapists with more years of experience in the field generally reported more varied use of treatment components, both overall at any given time and across time, that is, across the four quarters of the 12-month study period. Further, in some cases and at certain times (i.e., the last quarter of the study period), more experienced therapists reported using certain treatment components less than less experienced therapists such as infant-parent psychotherapy. This variability does not necessarily indicate less fidelity to the model; instead, it is possible that variability seen among more experienced therapists is indicative of more flexible adaptation of the model to individual families based on everchanging needs and priorities in the treatment. Indeed, others have noted in the literature that more experienced clinicians working in community practice settings make greater attempts to individualize or adapt treatment models to fit client needs, which leads to better outcomes (Berkel et al., 2011). More experienced therapists may also recognize that flexibility and responsivity to in-the-moment, and often unexpected, needs and issues are necessary to sustain the therapeutic relationship; as a result, they may be more likely to prioritize such over a planned agenda or curriculum expectation. This is consistent with the notion that flexibility is, in fact, an indicator of fidelity for relationally oriented therapies (e.g., CPP; Lieberman et al., 2015) and that "flexibility within fidelity" is optimal for achieving the best clinical intervention outcomes (Kendall \& Frank, 2018). As long as such adaptations do not interfere with the provision of model key ingredients, 
prior findings suggest that such flexibly applied treatments are associated with lower dropout rates and better outcomes (Forgatch, Patterson \& DeGarmo, 2005).

There are other possible reasons for the relatively less frequent (albeit still quite high) endorsement of some core components by more experienced clinicians at certain times during the study period. It is possible that more experienced clinicians may "drift" from using the core elements of the model over time, perhaps as they gain confidence in their abilities and/or accumulate more varied therapy experiences (e.g., with other populations or treatment models). If so, findings suggest that experienced therapists might benefit from continued supervision and support specific to implementing core components of the IMH-HV model. Of course, it is also possible that more experienced clinicians deliver the model to fidelity as much as less experienced clinicians, but relied less on formally indicating therapeutic strategies or techniques on the fidelity forms in the current study; instead, relying on an internal sense of "knowing" what they are doing. Still further, it is possible that more experienced clinicians could better assess what strategies were or were not helping families and adapted their techniques accordingly later in treatment as a result. Future studies that incorporate both self-report and observational measures of fidelity could test these possibilities to further clarify practice differences between more and less experienced therapists.

Another important finding in the present study was that there was more variability of reported treatment strategies in general and over the 12-month study period when working with higher risk families as compared to lower risk families, as defined by a number of demographic and psychological risk characteristics. In contrast, some studies have found greater endorsement of treatment components (i.e., greater fidelity in this sense) among therapists working with parents who are more impaired (Forgatch et al., 2005); thus, findings appear to be mixed at this point in time. Similar to speculations given above regarding the current set of results with more (vs. less) experienced therapists, it is likely that therapists in this study demonstrated more flexibility in their adaptation of the model and greater responsivity to families with ever-changing needs and more instability in their circumstances. If so, it is likely that such flexibility when working with higher risk families, specifically, benefits the therapeutic relationship, and ultimately, the treatment outcomes (Lieberman et al., 2015; Suchman et al., 2012). It will be important to examine this in future studies.

Several unexpected findings are worthy of further consideration here as well. First, results indicated that therapists, overall, reported little use of videotaping in the home, with even less reporting of using video review with families. Findings indicated that less experienced therapists used videotaping less frequently than more experienced therapists, although both groups showed relatively low rates, as well as a substantial drop in use of video across time (e.g., less experienced therapists reported video use only $18 \%$ of the time and more experienced therapists reported use of videotaping $27 \%$ of the time in the fourth quarter). These low rates were surprising given that the IMH-HV model includes use of video as an important treatment strategy (albeit more recently, see Rosenblum et al., this issue), and past research has shown that video feedback reduces maternal negative attributions of the child and improves reflective functioning and observed maternal sensitivity (Rosenblum et al., this issue; Sealy \& Glovinsky, 2016; Schechter et al., 2006; Steele et al., 2014). These results indicate that IMH-HV therapists may need more training and support for use of video during sessions with families, especially newer therapists in the field. Alternatively, the low rates of video use may reflect certain barriers to video use that IMH therapists faced such as a high case load or inconsistent availability of video recording equipment.

Another surprising finding was the low rates of "planning for termination" among all therapists across the study duration; the low rate of termination planning in the later stages of therapy was especially surprising, as was the fact that less experienced therapists showed a "decline" in termination planning over time (with only 5\% of sessions in the fourth quarter noting this strategy among less experienced therapists). In contrast, and more along the lines of what would be expected, more experienced therapists reported a notable "increase" in termination planning near the end of the study period, albeit still infrequently reported (11\% of sessions). It is important to note that in the current study, there were very few planned terminations; across the 12-month study period only six of the $78(7.7 \%)$ participating families had a planned therapeutic ending due to "goals being met" according to the therapist. For those families, termination planning was used in $42 \%$ of sessions in the quarter prior to the end of treatment. Thus, it is possible that therapists were not discussing termination with families because they were expecting the treatment relationship to continue. On the flip side, it is possible that families dropped out of treatment due, in part, to not fully understanding the therapeutic "landscape" or trajectory because this was not discussed regularly with the therapist. That is, some families may have not felt that progress toward goals was being made or that there was an "end in sight" due to the infrequent discussion about the end of the treatment relationship. Thus, like the use of video during sessions, IMH-HV therapists might benefit from more training and support surrounding planning for and discussing termination with families, a clinical skill known to be very difficult. It may be especially imperative to emphasize the importance of termination discussions with families "well before" goals have been met, knowing that many families will not be able to sustain receiving services and may, unexpectedly, drop out of treatment. Indeed, unexpected, premature treatment dropout is common in community-delivered home visiting services with high-risk 
families and not unique to the IMH-HV model (Boller et al., 2014; Brand \& Jungmann, 2014; Foulon et al., 2015).

Despite these and other surprising findings, overall, results from this study demonstrate that IMH-HV therapists used a range of treatment strategies that varied somewhat by therapist years of experience and family risk status. Further, IMH-HV therapists utilized developmental guidance and infant-parent psychotherapy (two of the model's core components) most frequently and viewed these as among the most significant, if not the most significant, interventions during treatment sessions with families. In fact, rates of these two core components were well above the fidelity rates $(\sim 60 \%)$ suggested by implementation researchers as being the level at which fidelity begins to more strongly improve treatment outcomes (Durlak \& Dupree, 2008). Although utilization of a number of other treatment strategies were reported to occur at lower to moderate rates, it is reasonable to expect this would be the case given that it is impossible (and possibly not effective) to do many different things during single treatment sessions. Indeed, therapists in this study reported that they used, on average, 4.5 treatment strategies during individual sessions that lasted, on average, about $1.5 \mathrm{hr}$, which anecdotally seems to be a reasonable amount to try to accomplish during single IMH-HV sessions.

As in all studies, this one had both notable strengths and important limitations to consider. Results from this study add to a sparse, but growing, body of implementation literature that details how clinicians are practicing in community (not laboratory/research) settings. Implementation research on early childhood mental health home visiting models is even scarcer. Although the sample size of therapists and participating families was somewhat small, the sample represented IMH-HV therapists with a range of experience and included a size-able group of more novice therapists, which better represents the workforce in this field as compared to intervention trials only utilizing "expert" or very experienced therapists. Furthermore, the sample was drawn from 12 different mental health agencies across both rural and urban settings, again better representing "real-world" work in the field. An additional strength of the study was the examination of fidelity among important subgroups within the full sample, specifically, groups based on years of experience and groups based on family risk status. This is important because there is natural variability among providers of any intervention model, and reporting fidelity or other implementation factors for an entire sample only may obscure meaningful differences between more or less effective clinicians (Durlak \& Dupree, 2008). Finally, an important strength of this study was the use of clinician reports on fidelity "over multiple time periods for treatment with specific families," rather than obtaining reports on fidelity just once or for clinical practice "in general." This allowed us to examine trajectories, or stability and change, in fidelity over time as treatments unfolded from initial evalu- ations to ongoing therapeutic work to, in some cases, the end of the therapeutic relationship.

Although results provided important information about fidelity, the fidelity checklist created for this study had some notable limitations. First, it measured only one specific aspect of implementation-fidelity as operationalized by frequency of delivered treatment strategies central to the IMH-HV model. Other important aspects of implementation were not examined such as the quality of delivery or specific adaptations or alterations of the model made by therapists, both of which are known to impact outcomes (Berkel et al., 2011). Also, this study relied on therapists' self-reported fidelity, or adherence, to the model without any more objective (e.g., coded videotaped sessions) measures of fidelity. Although objective ratings of fidelity may be ideal, they are quite difficult to achieve for an intervention model as flexible and responsive to family needs as this one is. Indeed, other effective early childhood home visiting models that emphasize flexibility, such as CPP, also utilize clinicians' own ratings of fidelity, which are, in some cases, discussed during supervision (e.g., David \& Schiff, 2018; Lowell, Carter, Godoy, Paulicin, \& Briggs-Gowan, 2011). Nevertheless, future research would benefit from having both self-reported and observer-rated measures of fidelity to compare and contrast and to link with therapeutic outcomes; this is, in fact, just being undertaken by the present research team through a randomized clinical trial of the IMH-HV model in Michigan. Finally, as noted earlier, therapists were asked to mark which of the 15 broad categories of intervention strategies that they used and were not required to mark more specific techniques used within each category; specific examples within each category were provided simply for consideration and to help operationalize each category. As a result, use of more specific techniques could not be analyzed, for instance, different techniques within infant-parent psychotherapy that were more aligned with exploration of "ghosts in the nursery" versus exploration of current thoughts and feelings about the child without links back to caregiver history. The use of broad categories only and not more specific techniques on the fidelity checklist may have contributed to some of the current results such as the very high endorsed use of infant-parent psychotherapy, as noted earlier.

In conclusion, findings from the current study help to explicate IMH-HV model practices in the state of Michigan, where IMH-HV has been delivered for decades across the entire state. Results show that therapists are practicing with a high level of fidelity to a number of core intervention strategies or components of the model, at least according to their own self-reports, and also practice in a seemingly flexible manner using varied techniques during individual sessions and over time with families they serve. Findings also reveal important differences between meaningful groups of therapists and families, which emphasize the need to continue examining fidelity 
within and across different therapists with varying levels of training and experience. Importantly, results provide important implications for the training of IMH-HV therapists at different levels of experience. Current training and ongoing reflective supervision appear to strongly support therapist use of several core components of the model (namely, developmental guidance, infant-parent psychotherapy, and provision of emotional support), but more training may be needed for effective use of other treatment strategies. Specific training aimed at the key elements of the IMH-HV model may be especially critical to enhance fidelity to the model per se versus IMH principles that may apply more broadly to clinical practice across different types of treatments. It is possible that increasing therapist use of treatment strategies that are central to the model, but rarely used, through specific trainings and reflective supervision will strengthen the quality of services even further to better serve high-need families in the IMHHV population.

\section{ACKNOWLEDGMENTS}

The authors gratefully acknowledge that this research was made possible through the financial support provided by the Michigan Department of Health and Human Services, the Ethel and James Flinn Foundation, the Gerstacker Foundation, the University of Michigan's Women and Infants Mental Health Program, and the Michigan Department of Health and Human Services Children's Mental Health Block Grant (K. Rosenblum and M. Muzik, PIs).

\section{REF E R EN CES}

Beam, RJ, O'Brien, R. A., \& Neal, M. (2010). Reflective practice enhances public health nurse implementation of nurse-family partnership. Public Health Nursing, 27(2), 131-139. https://doi.org/ 10.1111/j.1525-1446.2010.00836.x

Berkel, C., Mauricio, Am. M., Schoenfelder, E., \& Sandler, I. N. (2011). Putting the pieces together: An integrated model of program implementation. Prevention Science, 12, 23-33. https://doi.org/ 10.1007/s11121-010-0186

Boller, K., Daro, D., Del Grosso, P., Cole, R., Paulsell, D., Hart, B., .. Hargreaves, M., (2014). Making replication work: Building infrastructure to implement, scale-up, and sustain evidence-based early childhood home visiting programs with fidelity. Princeton, NJ: Mathematica Policy Research.

Brand, T. \& Jungmann, T. (2014). Participant characteristics and process variables predict attrition from a home-based early intervention program. Early Childhood Research Quarterly, 29, 155-167. https://doi.org/10.1016/j.ecresq.2013.12.001

Caron, E. B., Bernard, K. \& Dozier, M. (2016). In vivo feedback predicts parent behavior change in the attachment and biobehavioral catch-up intervention. Journal of Clinical Child and Adolescent Psychology, 47, S35-S46. https://doi.org/10.1080/15374416.2016.1141359

Casillas, K.L., Fauchier, A., Derkash, B. T., \& Garrido, E. F. (2016). Implementation of evidence-based home visiting programs aimed at reducing child maltreatment: A meta-analytic review. Child Abuse \& Neglect, 31(8), 829-852. https://doi.org/10.1016/ j.chiabu.2015.10.009

Dane, A. V. \& Schneider, B. H. (1998). Program integrity in primary and early secondary prevention: Are implementation effects out of control? Clinical Psychology Review, 18(1), 23-45. https://doi.org/10.1016/S0272-7358(97)00043-3

David, P. \& Schiff, M. (2018). Initial clinician reports of the bottom-up dissemination of an evidence-based intervention for early childhood trauma. Child Youth Care Forum, 47, 115-132. https://doi.org/10.1007/s10566-017-9422-1

Derzon, J. H., Sale, E., Springer, J. F., \& Brounstein, P. (2005). Estimating intervention effectiveness: Synthetic projection of field evaluation results. The Journal of Primary Prevention, 26(4), 321-343. https://doi.org/10.1007/s10935-005-5391-5

Domitrovich, C. E. \& Greenberg, M. T. (2000). The study of implementation: Current findings from effective programs that prevent mental disorders in school-aged children. Journal of Educational and Psychological Consultation, 11(2), 193-221. https://doi.org/ 10.1207/S15327868XJEPC110204

DuBois, D. L., Holloway, B. E., Valentine, J.C., \& Cooper, H. (2002). Effectiveness of mentoring programs for youth: A meta-analytic review. American Journal of Community Psychology, 30(2), 157198. https://doi.org/10.1023/A:1014628810714

Durlak, J. A. \& Dupree, E. P. (2008). Implementation matters: A review of research on the influence of implementation on program outcomes and other factors affecting implementation. American Journal of Community Psychology, 41, 327-350. https://doi.org/ 10.1007/s10464-008-9165-0

Eames, C., Daley, D., Hutchings, J., Whitaker, C. J., Jones, K., Hughes, J. C., \& Bywater, T. (2009). Treatment fidelity as a predictor of behavior change in parents attending group-based parent training. Child: Care, Health and Development 35(5), 603-612. https://doi.org/10.1111/j.1365-2214.2009.00975.z

Felitti, V. J., Anda, R. F., Nordenberg, D., Williamson, D. F., Spitz, A. M., Edwards, V., ... Marks, J. S. (1998). Relationship of childhood abuse and household dysfunction of the leading causes of death in adults. The Adverse Childhood Experiences ACE Study. American Journal of Preventative Medicine, 14, 245-258.

Fixsen, D. L., Naoom, S. F., Blase, K. A., Friedman, R. M. \& Wallace, F. (2005). Implementation research: A synthesis of the literature. Tampa: FL: University of South Florida, Louis de la Parte Florida Mental Health Institute, The National Implementation Research Network (FMHI Publication \#231).

Forgatch, M. S., Patterson, G. R. \& DeGarmo, D. S. (2005). Evaluating fidelity: Predictive validity for a measure of competent adherence to the Oregon Model of Parent Management Training. Behavioral Therapy, 36(1), 3-13. https://doi.org/10.1016/S00005-7894)05)80049-8

Foulon, S., Greacen, T., Pasquet, B., Dugravier, R., Saias, T., Guedeney, N., ... Group, C. S. (2015). Predictors of study attrition in a randomized controlled trial evaluating a perinatal home-visiting program with mothers with psychosocial vulnerabilities. PLoS One, 10, e0142495. https://doi.org/10.1371/journal.pone.0142495

Fraiberg, S. (1980). Clinical studies in infant mental health: the first year of life. New York, NY: Basic Books.

Hulleman, C. S., \& Cordray, D. S. (2009). Moving from the lab to the field: The role of fidelity and achieved relative intervention strength. Journal of Research on Educational Effectiveness, 2, 88110. https://doi.org/10.1080/19345740802539325 
Kendall, P. C. \& Frank, H. E. (2018). Implementing evidence-based protocols: Flexibility within fidelity. Clinical Psychology Science and Practice, 25, 1-12. https://doi.org/10.1111/cpsp.12271

Lieberman, A. F., Ghosh Ippen, C., \& Van Horn, P. (2015). Don't hit my mommy! A manual for child-parent psychotherapy with young children exposed to violence and other trauma (2nd ed.). Washington, DC: Zero to Three.

Lowell, D. L., Carter, A. S., Godoy, L., Paulicin, B., \& BriggsGowan, M. J. (2011). A randomized controlled trial of Child FIRST: A comprehensive home-based intervention translating research into early childhood practice. Child Development, 82, 193-208. https://doi.org/10.1111/j.1467-8624.2010.01550.x

Michigan Association for Infant Mental Health. (2018). IMH Endorsement ${ }^{\circledR}$ Requirements/Guidelines. Retrieved from https://mi-aimh. org/endorsement/requirements/

Perepletchivoka, F., Treat, T. A., \& Kazdin A. E. (2007). Treatment integrity in psychotherapy research: Analysis of the studies and examination of the associated factors. Journal of Consulting and Clinical Psychology, 75(6), 829-841. https://doi.org/10.1037/ 0022-006X.75.6.829

Roben, C. K. P., Dozier, M., Caron, E. B., \& Bernard, K. (2017). Moving and evidence-based parenting program into the community. Child Development, 88(5), 1447-1452. https://doi.org/10.1111/ cdev.12898.

Rosenblum, K. L., Muzik, M., Jester, J., Huth-Bocks, A., Erickson, N., Ludtke, M., ... the Michigan Collaborative for Infant Mental Health Research. (this issue). Community delivered publicallyfunded infant-parent psychotherapy improves sensitive caregiving: Evaluation of the Michigan model of infant mental health home visiting. Infant Mental Health Journal.

Schechter, D. S., Myers, M. M., Brunelli, S. A., Coates, S. W., Zeanah, C. H., Mark Davies, ... Liebowitz, M. R. (2006). Traumatized mothers can change their minds about their toddlers: Understanding how a novel use of videofeedback supports positive change of maternal attributions. Infant Mental Health Journal, 27(5), 429-447. https://doi.org/10.1002/imhj.20101

Sealy, J. \& Glovinsky, I. P. (2016). Strengthening the reflective functioning capacities of parents who have a child with a neurodevelopmental disability through a brief, relationship-focused intervention. Infant Mental Health Journal, 37(2), 115-124. https://doi.org/10.1002/imhj.21557.

Shea, S. E., Jester, J. M., Huth-Bocks, A. C., Weatherston, D. J., Muzik, M., \& Rosenblum, K. L. (2020). Infant mental health home visiting therapists' reflective supervision self-efficacy in community practice settings. Infant Mental Health Journal, 41(2), 191-205.

Smith, J. D., Schneider, B. H., smith, P. K., \& Ananiadou, K. (2004). The effectiveness of whole-school antibullying programs: A synthesis of evaluation research. School Psychology Review, 33(4), 547-560.

Steele, M., Steele, H., Bate, J., Knafo, H., Kinsey, M., Bonuck, K., Meisner, P. \& Murphy, A. (2014). Looking from the outside in: the use of video in attachment-based interventions. Attachment and Human Development, 16(4), 402-415. https://doi.org/10.1080/ 14616734.2014.912941

Suchman, N. E., Decoste, C., Rosenberger, P., \& McMahon, T. J. (2012). Attachment-based intervention for substance-using mothers: A preliminary test of the proposed mechanisms of change. Infant Mental Health Journal, 33(4), 360-371. https://doi.org/10.1002/imhj.21311

Weatherston, D., Ribaudo, J., \& the Michigan Collaborative for Infant Mental Health. (this issue). Infant mental health home visiting: The evolution of a model. Infant Mental Health Journal.

Weatherston, D., \& Tableman, B. (2015). Infant mental health home visiting: Supporting competencies/reducing risks (3rd ed.). Southgate MI: Michigan Association for Infant Mental Health.

Weatherston, D., Tableman, B., Ludtke, M., Muzik, M. \& Rosenblum, K.R. (2016). Infant mental health-home visiting fidelity tool, version 3. Unpublished Manuscript.

Webster Stratton (1990). The Incredible Years: A trouble shooting guide for parents of children aged 3-8. Umbrella Press.

Weisz, J. R., \& Jensen P. S, (1999). Efficacy and effectiveness of child and adolescent psychotherapy and pharmacotherapy. Mental Health Service Research, 1(3), 125-157. https://doi.org/10.1023/ A:1022321812352.

How to cite this article: Huth-Bocks AC, Jester JM, Stacks AM, Muzik M, Rosenblum KL, the Michigan Collaborative for Infant Mental Health Research. Infant mental health home visiting therapists' fidelity to the Michigan IMH-HV model in community practice settings. Infant Ment Health J. 2020;41:206-219. https://doi.org/10.1002/imhj.21839 